\title{
Uma aplicação da dinâmica de uma partícula em uma trajetória predeterminada: o problema do iglu elipsoidal
}

An application of the dynamics of a particle in a predetermined path: the problem of the ellipsoidal igloo

\author{
R. S. Dutra*10, L. C. Ribeiro², C. M. Porto ${ }^{3}$ \\ ${ }^{1}$ Instituto Federal de Educação, Ciência e Tecnologia, Paracambi, RJ, Brasil \\ ${ }^{2}$ Centro Federal de Educação Tecnológica Celso Suckow da Fonseca, Nova Iguaçu. RJ, Brasil \\ ${ }^{3}$ Universidade Federal Rural do Rio de Janeiro, Departamento de Física, Seropédica, RJ, Brasil
}

Recebido em 19 de Junho, 2018. Revisado em 06 de Setembro, 2018. Aceito em 10 de Setembro, 2018.

\begin{abstract}
Neste trabalho o problema clássico de uma partícula que descreve um arco de círculo ao deslizar pela superfície polida de um iglu esférico é estendido para o caso de um iglu elipsoidal. Neste caso, a altura na qual a partícula perde contato com a superfície do iglu passa a obedecer a uma equação cúbica com coeficientes que dependem dos parâmetros que determinam a trajetória elíptica. A solução analítica dessa equação é então obtida a partir do método de Cardano-Tartaglia.
\end{abstract}

Palavras-chave: Mecânica clássica; Movimento bidimensional; Trajetória predeterminada.

In the present work the classical problem of a particle which describes a segment of a circular path while sliding over a polished spherical surface of an igloo is extended to the case of an ellipsoidal igloo. In this case, the height in which the particle loses the contact with the surface of the igloo obeys a cubic equation whose coefficients depends on the parameters that determine the elliptical path. The analytical solution of this equation is then obtained through the method of Cardano-Tartaglia.

Keywords: Classical mechanics; Two-dimensional motion; Predetermined path.

\section{Introdução}

O problema de uma partícula abandonada do topo de uma superfície esférica perfeitamente polida é frequentemente abordado na disciplina de mecânica newtoniana ministrada nos primeiros períodos dos cursos de graduação das carreiras da área de ciências exatas, no contexto do estudo da conservação da energia mecânica de sistemas físicos. A título de ilustração, os livros didáticos apresentam o caso de um iglu modelado por uma semiesfera polida de raio $R$. A conservação da energia mecânica é então considerada entre o instante em que a partícula é abandonada do topo do iglu, quando a energia mecânica total do sistema, $E=U+K$, se resume à energia potencial $U=m g R$, sendo $m$ a massa da partícula e $g$ a aceleração da gravidade local, e o momento em que ela perde o contato com a superfície do iglu, a uma altura h, quando a energia mecânica do sistema é dada por $E=m g h+\frac{1}{2} m v^{2}$, sendo $v$ a velocidade da partícula. A altura $h$ na qual a partícula perde contato com a superfície é então obtida pelo princípio da conservação da energia mecânica, resultando em $h=\frac{2 R}{3} \sqrt{1}, 2$, resultado este que depende do parâmetro $R$, responsável por determinar a trajetória descrita pela partícula até o momento em que ela perde o contato com a superfície. O interesse

*Endereço de correspondência: rafael.dutra@ifrj.edu.br nesse problema está na possibilidade de sua extensão para casos em que a partícula desliza por superfícies de geometrias distintas, como na situação na qual ela descreve uma trajetória bidimensional elíptica, determinada pelos parâmetros $a$ e $b$ (semieixos da elipse), ao deslizar pela superfície de um iglu elipsoidal.

Na segunda seção deste trabalho apresentamos um estudo rigoroso da cinemática de uma partícula que desliza por uma superfície de geometria predeterminada, descrevendo uma trajetória bidimensional associada ao perfil dessa superfície. Para isso, utilizamos elementos básicos de geometria diferencial, como o raio de curvatura e os vetores unitários tangente e normal à trajetória da partícula 3 5. O estudo apresentado tem validade geral e pode ser aplicado ao movimento de uma partícula que descreve uma trajetória bidimensional qualquer ao deslizar por uma superfície perfeitamente polida. $\mathrm{Na}$ terceira seção consideramos a partícula descrevendo uma trajetória bidimensional elíptica associada ao perfil da superfície perfeitamente polida de um iglu elipsoidal, representado geometricamente pelo hemisfério superior de um elipsoide de revolução. Nessa seção estudamos a dinâmica do sistema, considerando as forças newtonianas que atuam sobre a partícula, no caso, as forças peso e normal de contato. A quarta e última seção é dedicada às considerações finais. 


\section{Cinemática bidimensional}

A posição de uma partícula que descreve uma trajetória genérica $C$ no plano $x y$ é determinada pelo vetor

$$
\mathbf{r}(t)=x(t) \hat{\mathbf{i}}+y(t) \hat{\mathbf{j}}
$$

onde $\hat{\mathbf{i}}$ e $\hat{\mathbf{j}}$ são os vetores unitários associados aos eixos cartesianos $x$ e $y$, respectivamente. As coordenadas cartesianas da partícula, $x(t)$ e $y(t)$, são parametrizadas pelo tempo $t$ e estão submetidas a um determinado vínculo $y=f(x)$, responsável por definir a própria trajetória $C$. Considerando o vetor posição $\mathbf{r}(t)$ parametrizado em termos do comprimento de arco $s$, podemos, com o auxílio da regra da cadeia, escrever o vetor velocidade

$$
\mathbf{v}(t)=\frac{d \mathbf{r}}{d s} \frac{d s}{d t}=v \frac{d \mathbf{r}}{d s}
$$

onde $v=d s / d t$ corresponde a componente tangencial da velocidade instantânea da partícula.

O elemento de linha $d \mathbf{r}$, por sua vez, é definido por

$$
d \mathbf{r}=d x \hat{\mathbf{i}}+d y \hat{\mathbf{j}}
$$

e possui módulo

$$
d s=|d \mathbf{r}|=\sqrt{1+y^{\prime}(x)^{2}} d x,
$$

sendo $y^{\prime}(x)=d y / d x$. Dividindo a equação (3) pela equação (4) obtemos o vetor unitário $\hat{\mathbf{t}}$ tangente à trajetória da partícula,

$$
\hat{\mathbf{t}}=\frac{d \mathbf{r}}{d s}=\frac{\hat{\mathbf{i}}+y^{\prime}(x) \hat{\mathbf{j}}}{\sqrt{1+y^{\prime}(x)^{2}}} .
$$

Derivando a equação (2) em relação ao tempo e considerando a equação (5), obtemos, para o vetor aceleração da partícula,

$$
\mathbf{a}(t)=\frac{d^{2} s}{d t^{2}} \hat{\mathbf{t}}+\frac{d s}{d t} \frac{d \hat{\mathbf{t}}}{d t}=\frac{d^{2} s}{d t^{2}} \hat{\mathbf{t}}+v \frac{d \hat{\mathbf{t}}}{d t} .
$$

A derivada do vetor unitário $\hat{\mathbf{t}}$ em relação ao tempo, por sua vez, pode ser escrita como

$$
\frac{d \hat{\mathbf{t}}}{d t}=\frac{d \hat{\mathbf{t}}}{d s} \frac{d s}{d t}=v \frac{d \hat{\mathbf{t}}}{d s} .
$$

Já a derivada desse vetor em relação ao comprimento de $\operatorname{arcos} s$ é dada por

$$
\frac{d \hat{\mathbf{t}}}{d s}=\frac{d \hat{\mathbf{t}}}{d x} \frac{d x}{d s}
$$

onde

$$
\frac{d x}{d s}=\frac{1}{\sqrt{1+y^{\prime}(x)^{2}}}
$$

e

$$
\frac{d \hat{\mathbf{t}}}{d x}=-\frac{y^{\prime \prime}(x)}{\left(1+y^{\prime}(x)^{2}\right)^{3 / 2}}\left(y^{\prime}(x) \hat{\mathbf{i}}-\hat{\mathbf{j}}\right) .
$$

Substituindo as equações (9) e (10) na equação (8) e levando o resultado na equação (7) obtemos

$$
\frac{d \hat{\mathbf{t}}}{d t}=-\frac{y^{\prime \prime}(x) v}{\left(1+y^{\prime}(x)^{2}\right)^{2}}\left(y^{\prime}(x) \hat{\mathbf{i}}-\hat{\mathbf{j}}\right),
$$

que, substituída na equação (6), fornece o vetor aceleração a escrito em termos dos vetores unitários $\hat{\mathbf{t}}$ e $\hat{\mathbf{n}}[2]$,

$$
\mathbf{a}=\frac{d^{2} s}{d t^{2}} \hat{\mathbf{t}}+\frac{v^{2}}{\rho} \hat{\mathbf{n}}
$$

sendo

$$
\hat{\mathbf{n}}=\frac{d \hat{\mathbf{t}}}{d s} /\left|\frac{d \hat{\mathbf{t}}}{d s}\right|=\frac{\left(-y^{\prime}(x) \hat{\mathbf{i}}+\hat{\mathbf{j}}\right)}{\sqrt{1+y^{\prime}(x)^{2}}} \times \operatorname{sgn}\left(y^{\prime \prime}(x)\right)
$$

o vetor unitário normal à trajetória da partícula e

$$
\rho=\frac{\left(1+y^{\prime}(x)^{2}\right)^{3 / 2}}{\left|y^{\prime \prime}(x)\right|},
$$

o raio de curvatura em um determinado ponto dessa trajetória. As grandezas $d^{2} s / d t^{2}$ e $v^{2} / \rho$ representam, respectivamente, as componentes tangencial e normal (ou centrípeta) do vetor aceleração da partícula. É importante mencionar o caráter geral da expressão 12 obtida para o vetor aceleração. Essa expressão pode ser aplicada ao problema de uma partícula que desliza sem atrito sobre uma superfície de geometria qualquer, descrevendo uma trajetória com raio de curvatura $\rho$ definido pela expressão (14). Na seção a seguir apresentamos uma aplicação ao problema de uma partícula que desliza sobre a superfície perfeitamente polida de um iglu elipsoidal representado geometricamente pelo hemisfério superior de um elipsóide de revolução.

\section{O problema do iglu}

Consideramos inicialmente uma partícula que desliza sobre uma superfície genérica perfeitamente polida, sob a ação da força peso $\mathbf{P}=-m g \hat{\mathbf{j}}$ e da força normal $\mathbf{N}$ aplicada pela superfície ao longo do trajeto de comprimento s. A partir da segunda lei de Newton $\left(\mathbf{F}_{R}=\mathbf{P}+\mathbf{N}=m \mathbf{a}\right.$, onde $\mathbf{F}_{R}$ é a resultante das forças que atuam na partícula de massa $m$ ) obtemos que a dinâmica da partícula é descrita pela equação de movimento

$$
\mathbf{N}+\mathbf{P}=m \frac{d^{2} s}{d t^{2}} \hat{\mathbf{t}}+\frac{m v^{2}}{\rho} \hat{\mathbf{n}},
$$

na qual utilizamos o vetor aceleração da partícula definido pela expressão 12 .

A equação 15 descreve a dinâmica de uma partícula que desliza sem atrito sobre uma superfície qualquer, efetuando uma trajetória bidimensional determinada pelos vetores unitários $\hat{\mathbf{t}}$ e $\hat{\mathbf{n}}$ e pelo raio de curvatura $\rho$, dados, respectivamente, pelas equações (5), (13) e (14). Essa 
equação pode ser decomposta em uma componente com projeção na direção do vetor unitário $\hat{\mathbf{t}}$,

$$
\mathbf{P} \cdot \hat{\mathbf{t}}=m \frac{d^{2} s}{d t^{2}}
$$

e outra componente com projeção na direção do vetor unitário $\hat{\mathbf{n}}$,

$$
-N+\mathbf{P} \cdot \hat{\mathbf{n}}=\frac{m v^{2}}{\rho} .
$$

A componente tangencial do vetor aceleração, dada pela derivada segunda do comprimento de arco $s$ em relação ao tempo, $\frac{d^{2} s}{d t^{2}}$, pode ser escrita em termos da coordenada $y$ e de suas derivadas temporais,

$$
\begin{aligned}
\frac{d s}{d t} & =\sqrt{1+x^{\prime}(y)^{2}} \dot{y} \rightarrow \frac{d^{2} s}{d t^{2}}=\frac{x^{\prime}(y) x^{\prime \prime}(y)}{\sqrt{1+x^{\prime}(y)^{2}}} \dot{y}^{2} \\
& +\sqrt{1+x^{\prime}(y)^{2}} \ddot{y} .
\end{aligned}
$$

Consideramos como aplicação uma partícula que descreve uma trajetória bidimensional elíptica ao deslizar sobre a superfície perfeitamente polida de um iglu elipsoidal geometricamente representado pelo hemisfério superior do sólido gerado pela rotação da elipse $\frac{x^{2}}{a^{2}}+\frac{y^{2}}{b^{2}}=1 \mathrm{em}$ torno do eixo cartesiano $y$, como mostra a figura 11. Essa partícula parte do repouso no topo do iglu e percorre um arco de comprimento $s$ até o momento em que perde contato com a superfície, a uma determinada altura $h$, medida em relação ao solo. Nesse caso, o vetor posição $\mathbf{r}$ pode ser parametrizado pela coordenada $y$ e escrito como

$$
\mathbf{r}= \pm a \sqrt{1-\frac{y^{2}}{b^{2}}} \hat{\mathbf{i}}+y \hat{\mathbf{j}}
$$

onde $a$ e $b$ são os semieixos do arco de elipse descrito pela partícula. $\mathrm{O}$ vetor unitário tangente $\hat{\mathbf{t}}$, parametrizado em termos do tempo $t$, é dado por

$$
\hat{\mathbf{t}}=\frac{d \mathbf{r}}{d t} /\left|\frac{d \mathbf{r}}{d t}\right| \text {. }
$$

Para a derivada $\frac{d \mathbf{r}}{d t}$ obtemos

$$
\frac{d \mathbf{r}}{d t}=\mp \frac{a y}{b \sqrt{b^{2}-y^{2}}} \frac{d y}{d t} \hat{\mathbf{i}}+\frac{d y}{d t} \hat{\mathbf{j}} .
$$

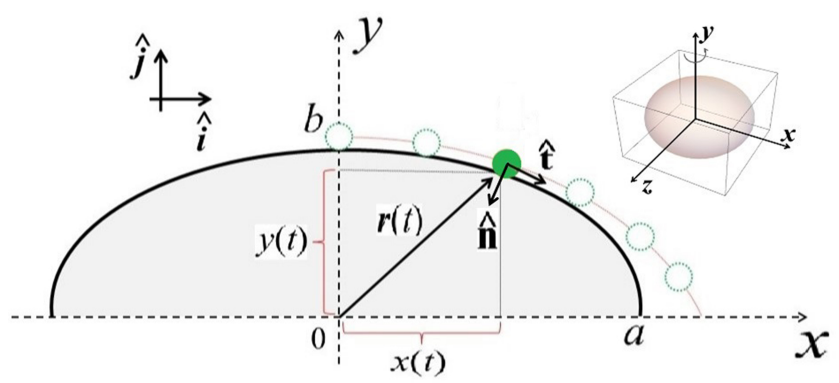

Figura 1: Trajetória bidimensional elíptica de uma partícula que desliza sobre a superfície perfeitamente polida de um iglu elipsoidal.
Retornando com essa expressão na equação 20, junto com a expressão para seu módulo, $\left|\frac{d \mathbf{r}}{d t}\right|$, e considerando o movimento da partícula como restrito ao primeiro quadrante do plano cartesiano, com $d y / d t<0$, uma vez que ela desliza para baixo sobre a superfície do iglu, obtemos que o vetor $\hat{\mathbf{t}}$ é dado pela expressão

$$
\hat{\mathbf{t}}=\frac{a y}{\sqrt{b^{4}+\left(a^{2}-b^{2}\right) y^{2}}} \hat{\mathbf{i}}-\frac{b \sqrt{b^{2}-y^{2}}}{\sqrt{b^{4}+\left(a^{2}-b^{2}\right) y^{2}}} \hat{\mathbf{j}} .
$$

O vetor normal $\hat{\mathbf{n}}$, por sua vez, é definido por

$$
\hat{\mathbf{n}}=\frac{d \hat{\mathbf{t}}}{d t} /\left|\frac{d \hat{\mathbf{t}}}{d t}\right| \text {. }
$$

Calculando a derivada de $\hat{\mathbf{t}}$ em relação ao tempo obtemos a expressão

$$
\begin{gathered}
\frac{d \hat{\mathbf{t}}}{d t}=\frac{b^{4} a}{\left(b^{4}+\left(a^{2}-b^{2}\right) y^{2}\right)^{3 / 2}} \frac{d y}{d t} \hat{\mathbf{i}} \\
+\frac{a^{3} b^{3} y}{\sqrt{b^{2}-y^{2}}\left(b^{4}+\left(a^{2}-b^{2}\right) y^{2}\right)^{3 / 2}} \frac{d y}{d t} \hat{\mathbf{j}} .
\end{gathered}
$$

Retornando com esse resultado na equação 23 junto com a expressão para o módulo $\left|\frac{d \hat{\mathbf{t}}}{d t}\right|$, obtemos

$$
\hat{\mathbf{n}}=-\frac{b \sqrt{b^{2}-y^{2}}}{\sqrt{b^{4}+\left(a^{2}-b^{2}\right) y^{2}}} \hat{\mathbf{i}}-\frac{a y}{\sqrt{b^{4}+\left(a^{2}-b^{2}\right) y^{2}}} \hat{\mathbf{j}}
$$

Considerando a equação (14) obtemos que o raio de curvatura $\rho$ para a trajetória elíptica é dado pela expressão

$$
\rho=\frac{\left(1+x^{\prime}(y)^{2}\right)^{3 / 2}}{\left|x^{\prime \prime}(y)\right|}=\frac{\left(b^{4}+\left(a^{2}-b^{2}\right) y^{2}\right)^{3 / 2}}{a b^{4}},
$$

na qual $x^{\prime}(y)$ e $x^{\prime \prime}(y)$ correspondem, respectivamente, às derivadas de primeira e segunda ordem da função

$$
x(y)=a \sqrt{1-y^{2} / b^{2}}
$$

obtida para o primeiro quadrante da elipse. A partir da equação 26 verificamos que o raio de curvatura $\rho$ da trajetória elíptica tende ao raio $R$ de um círculo no limite em que $a=b=R$. Calculando as derivadas $x^{\prime}(y)$ e $x^{\prime \prime}(y)$ a partir da equação (27), e substituindo os resultados na equação 18, obtemos

$$
\begin{aligned}
\frac{d^{2} s}{d t^{2}} & =\frac{b a^{2} y \dot{y}^{2}}{\left(b^{2}-y^{2}\right)^{3 / 2} \sqrt{b^{4}+\left(a^{2}-b^{2}\right) y^{2}}} \\
& +\frac{\sqrt{b^{4}+\left(a^{2}-b^{2}\right) y^{2}}}{b \sqrt{b^{2}-y^{2}}} \ddot{y} .
\end{aligned}
$$

O módulo da velocidade da partícula pode ser obtido de forma explícita em função da coordenada $y$, a partir do princípio da conservação da energia mecânica, 


$$
m g b=m g y+\frac{m v^{2}}{2} \rightarrow v=\sqrt{2 g(b-y)} .
$$

Substituindo na equação 16 as expressões obtidas para $\hat{\mathbf{t}}, \mathbf{P}$ e $d^{2} s / d t^{2}$, obtemos uma equação diferencial que descreve a dinâmica da coordenada $y$ em função dos semieixos $a$ e $b$ da elipse e da magnitude $g$ da aceleração da gravidade local,

$$
\left(b^{2}-y^{2}\right)\left(b^{4}+\left(a^{2}-b^{2}\right) y^{2}\right) \ddot{y}+b^{2} a^{2} y \dot{y}^{2}-g b^{2}\left(b^{2}-y^{2}\right)^{2}=0 .
$$

Da mesma forma, substituindo na equação 17 as expressões obtidas para $\mathbf{P}, \hat{\mathbf{n}}, \rho$ e $v$, obtemos uma expressão para o módulo $N$ da força normal que a superfície do iglu exerce sobre a partícula,

$$
N=m g\left(\frac{a y}{\sqrt{b^{4}+\left(a^{2}-b^{2}\right) y^{2}}}-\frac{2(b-y) a b^{4}}{\left(b^{4}+\left(a^{2}-b^{2}\right) y^{2}\right)^{3 / 2}}\right) .
$$

No caso particular de uma trajetória circular, na qual $a=b=R$, obtemos que a força normal apresenta uma dependência linear com a altura $y$, como mostrado no resultado da figura 2 (linha contínua). De fato, fazendo $a=b=R$ na equação (31), obtemos $N=3 m g y / R-2 m g$, expressão esta que fornece o clássico resultado $y=2 R / 3$ para a altura na qual a partícula perde contato com a superfície de um iglu de perfil esférico, quando $N=0$. A figura 2 apresenta ainda os resultados obtidos a partir da equação 31 nas situações em que $a<b$ (linha tracejada) e $a>b$ (linha pontilhada). Esses resultados mostram que a partícula perde contato mais rapidamente com a superfície do iglu na situação em que $a<b$, o que de

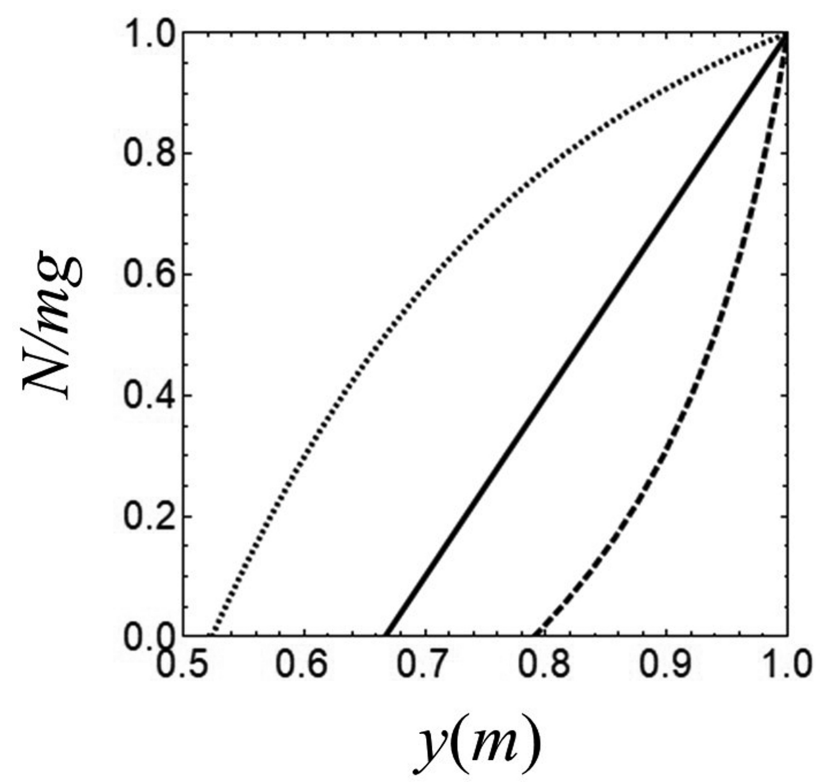

Figura 2: A figura mostra a razão entre as magnitudes $N$ da força de contato e $m g$ da força peso em função da altura $y$ da partícula. O semieixo $b$ é fixado em $b=1 \mathrm{~m}$. As curvas mostram os resultados obtidos para $a=2 b=2 m$ (linha pontilhada), $a=$ $b=1 m$ (linha contínua) e $a=b / 2=0.5 m$ (linha tracejada). certa forma já era intuitivamente esperado, uma vez que para um iglu mais estreito a partícula perde contato com a superfície em uma posição mais próxima de seu ponto de partida.

No caso geral de um iglu elipsoidal, o ponto onde a partícula perde contato é determinado pela solução da equação cúbica

$$
\left(a^{2}-b^{2}\right) y^{3}+3 b^{4} y-2 b^{5}=0,
$$

obtida ao fazermos $N=0$ na equação (31). Essa equação é do tipo $A y^{3}+B y^{2}+C y+D=0$ e pode ser resolvida de forma analítica a partir do método de Cardano-Tartaglia [6], que fornece as soluções

$y_{1}=-\frac{B}{3 A}+\sqrt[3]{-\frac{q}{2}+\sqrt{\frac{q^{2}}{4}+\frac{p^{3}}{27}}}+\sqrt[3]{\frac{q}{2}-\sqrt{\frac{q^{2}}{4}+\frac{p^{3}}{27}}}$,

$$
\begin{gathered}
y_{2}=-\frac{B}{3 A}+\left(-\frac{1}{2}+i \frac{\sqrt{3}}{2}\right) \sqrt[3]{-\frac{q}{2}+\sqrt{\frac{q^{2}}{4}+\frac{p^{3}}{27}}}+ \\
\left(-\frac{1}{2}-i \frac{\sqrt{3}}{2}\right) \sqrt[3]{\frac{q}{2}-\sqrt{\frac{q^{2}}{4}+\frac{p^{3}}{27}}}
\end{gathered}
$$

$$
\begin{gathered}
y_{3}=-\frac{B}{3 A}+\left(-\frac{1}{2}-i \frac{\sqrt{3}}{2}\right) \sqrt[3]{-\frac{q}{2}+\sqrt{\frac{q^{2}}{4}+\frac{p^{3}}{27}}}+ \\
\left(-\frac{1}{2}+i \frac{\sqrt{3}}{2}\right) \sqrt[3]{\frac{q}{2}-\sqrt{\frac{q^{2}}{4}+\frac{p^{3}}{27}}}
\end{gathered}
$$

$\operatorname{com} p=C / A-B^{2} / 3 A^{2}$ e $q=D / A-B C / 3 A^{2}+2 B^{3} / 27 A^{3}$, sendo, para o caso do iglu elipsoidal, $A=\left(a^{2}-b^{2}\right), B=0$, $C=3 b^{4}$ e $D=-2 b^{5}$. Retornando com essas constantes nas equações (33), 34 e 35), obtemos a solução

$$
y(a, b)= \begin{cases}f(a, b), & \text { se } a<b \\ g(a, b), & \text { se } a>b\end{cases}
$$

na qual

$$
\begin{aligned}
& f(a, b)=\frac{(1-i \sqrt{3})\left(a^{2} b^{4}-b^{6}\right)}{2\left(a^{2}-b^{2}\right)\left(a^{4} b^{5}-2 a^{2} b^{7}+b^{9}+Q(a, b)\right)^{1 / 3}}- \\
& \quad \frac{(1+i \sqrt{3})\left(a^{4} b^{5}-2 a^{2} b^{7}+b^{9}+Q(a, b)\right)^{1 / 3}}{2\left(a^{2}-b^{2}\right)} \\
& \mathrm{e}
\end{aligned}
$$

$$
\begin{aligned}
g(a, b)=- & \frac{a^{2} b^{4}-b^{6}}{\left(a^{2}-b^{2}\right)\left(a^{4} b^{5}-2 a^{2} b^{7}+b^{9}+Q(a, b)\right)^{1 / 3}}+ \\
& \frac{\left(a^{4} b^{5}-2 a^{2} b^{7}+b^{9}+Q(a, b)\right)^{1 / 3}}{a^{2}-b^{2}}
\end{aligned}
$$


onde

$$
Q(a, b)=\sqrt{a^{8} b^{10}-3 a^{6} b^{12}+3 a^{4} b^{14}-a^{2} b^{16}} .
$$

As soluções reais $g(a, b)$ e $f(a, b)$ correspondem respectivamente às raízes dadas pelas equações (33) e (34), após substituirmos nessas equações os coeficientes da equação cúbica 32 . Ressaltamos que para cada uma das regiões $(a<b$ e $a>b)$ obtivemos uma única raíz real, respectivamente dadas por $f(a, b)$ e $g(a, b)$, e duas outras complexas, para o problema em questão. A função $y(a, b)$ não é definida em $a=b$, o que justifica o intervalo aberto na equação 36 mas os limites laterais, por outro lado, existem e são iguais, $\lim _{a \rightarrow b} y(a, b)=\lim _{a \rightarrow b} f(a, b)=\lim _{a \rightarrow b} g(a, b) \rightarrow 2 b / 3$, recuperando o resultado clássico em que o elipsóide de revolução se degenera em uma esfera de raio $R=a=b$. Tais limites foram testados com o auxílio do software Mathematica e estão representados na figura 3 pelas linhas pontilhadas. $\mathrm{O}$ resultado apresentado nessa figura foi obtido a partir da equação 36 e fornece a altura $y$ na qual a partícula perde contato com a superfície do iglu em função do parâmetro $a$ associado à trajetória elíptica por ela descrita, com $b=1 \mathrm{~m}$. Tal gráfico corrobora o comportamento descrito na figura 2 mostrando um decaimento acentuado na magnitude da força normal $\mathbf{N}$ para $a<b$, como mencionado anteriormente. Através desse resultado observamos que a altura na qual a partícula perde contato decresce monotonicamente com o aumento do semieixo $a$. Por outro lado, quando esse parâmetro se aproxima de zero, obtemos que a partícula perde contato em uma posição mais próxima ao topo do iglu.

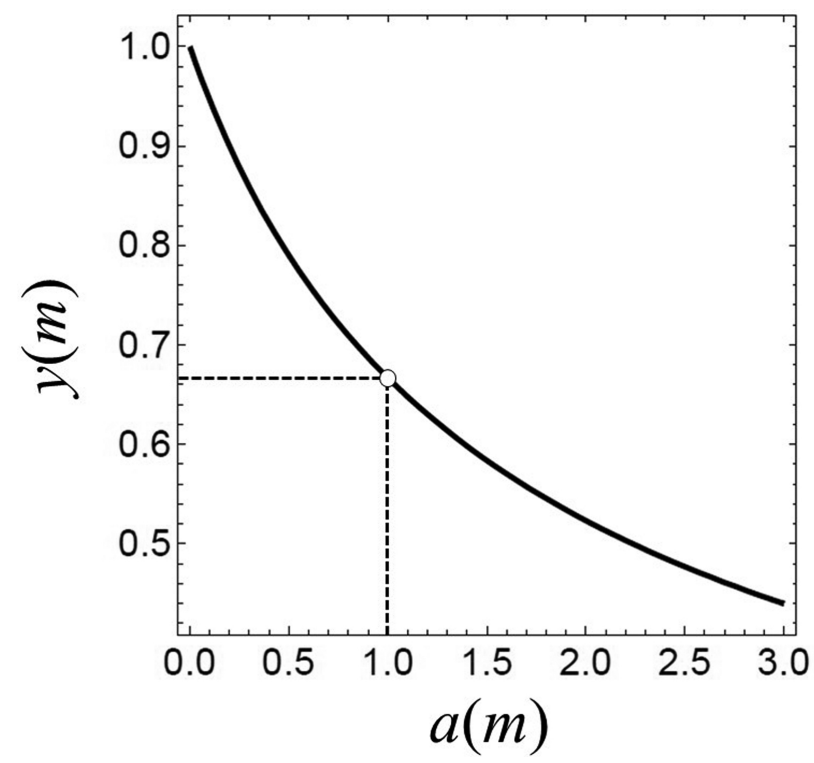

Figura 3: Altura $y$ na qual a partícula perde contato com a superfície do iglu em função do semieixo $a$ da elipse. O semieixo $b$ é fixado em $b=1 \mathrm{~m}$.

\section{Considerações Finais}

Neste trabalho estudamos a dinâmica do sistema composto por uma partícula que descreve uma trajetória bidimensional elíptica ao deslizar sem atrito pela superfície de um iglu elipsoidal representado geometricamente pelo hemisfério superior de um elipsóide de revolução. Como resultado obtivemos uma expressão analítica que fornece a magnitude $N$ da força normal de contato em função da altura $y$ da partícula e dos semieixos $a$ e $b$ da elipse associada à trajetória por ela descrita. A partir desse resultado, considerando $N=0$, chegamos a uma equação cúbica cuja solução, obtida a partir do método de Cardano-Tartaglia, fornece a altura na qual a partícula perde contato com a superfície do iglu. Além disso, apresentamos um desenvolvimento formal da cinemática bidimensional associada ao movimento de uma partícula que é posta a deslizar sobre uma superfície qualquer, descrevendo uma trajetória definida no plano $x y$ por uma determinada função $y=f(x)$. Tal desenvolvimento cumpre um papel pedagógico interessante por ser descrito em termos de elementos básicos de geometria diferencial, como os vetores unitários, normal e tangente à trajetória da partícula, e o próprio raio de curvatura, elementos estes muito pouco trabalhados na bibliografia tradicional, mas que se apresentam como ferramentas poderosas e que podem ser utilizadas na extensão do problema proposto neste trabalho a outros casos de interesse.

\section{Referências}

[1] D. Halliday, R. Resnick e J. Walker, Fundamentos de física: mecânica (LTC, Rio de Janeiro, 2009), v. 1, $8^{\text {a }}$ ed.

[2] H.M. Nussenzveig, Curso de física básica: mecânica (Edgar Bluncher, São Paulo, 2013), v. 1, 5 $5^{\mathrm{a}}$ ed.

[3] M.P. Carmo, Differential Geometry of Curves and Surfaces (Prentice-Hall, Englewood Cliffs, 1976).

[4] A.D. Pereira Jr e N.A. Lemos, Revista Brasileira de Ensino de Física 33, 2306 (2011).

[5] M. Alonso e E.J. Finn, Física: Um curso universitárioMecânica (Editora Blucher, São Paulo, 2018) v. 1.

[6] C.B. Boyer, História da Matemática (Edgar Bluncher, São Paulo, 1996). 